\title{
Comparison of effects of routine topical treatments in the milking parlor on digital dermatitis lesions
}

\author{
C. Jacobs,${ }^{* 1}$ K. Orsel, ${ }^{*}$ S. Mason, ${ }^{*} \dagger$ and H. W. Barkema* \\ *Department of Production Animal Health, University of Calgary, Calgary, Alberta, Canada T2N 4N1 \\ †Farm Animal Care Associates, Calgary, Alberta, Canada T2L 0T6
}

\begin{abstract}
Digital dermatitis (DD), an infectious bacterial disease affecting the feet of dairy cattle, can cause lameness and decrease milk production, fertility, and animal welfare. Current DD treatment typically involves routine hoof trimming and topical antibiotics. Several nonantibiotic commercial topical products are used for controlling DD lesions; however, there is limited or no evidence regarding their effectiveness. The objectives of this study were to evaluate 2 commercially available topical applications on their ability to (1) clinically cure active DD lesions to nonactive lesions and (2) prevent recurrence of active DD lesions. Ten farms were visited weekly. In the milking parlor, the hind feet of lactating cattle were cleaned and scored (M-stage scoring system). Cattle with DD lesions at the first visit were randomly allocated to 1 of 4 treatment groups: positive control (tetracycline solution), HealMax (AgroChem Inc., Saratoga Springs, NY), HoofSol (Diamond Hoof Care Ltd., Intracare BV, Veghel, the Netherlands), and a negative control (saline). All products were applied to lesions using a spray bottle. Tetracycline, HealMax, and HoofSol had a higher probability of clinical cure for active lesions compared with saline 1 wk after the first treatment (wk 1), with 69, 52, and $79 \%$ clinical cure of active lesions, respectively, compared with $34 \%$ with saline. At wk 7, the probability of clinical cure for active lesions was 10, 33, 31, and $45 \%$ of lesions treated weekly with saline, tetracycline, HealMax, and HoofSol, respectively (no difference among treatments). The substantial clinical cure with saline highlighted the potential importance of cleaning feet. In wk 1, treatment with saline, tetracycline, HealMax, and HoofSol resulted in a probability of recurrence of active DD lesions of $9,11,11$, and $8 \%$, respectively, with no product being superior to saline. After $7 \mathrm{wk}$, the probability
\end{abstract}

Received October 12, 2017.

Accepted February 14, 2018.

${ }^{1}$ Corresponding author: jacobs.casey@gmail.com of recurrence of active lesions was 5, 7, 6, and $6 \%$ for saline, tetracycline, HealMax, and HoofSol respectively, with no difference among groups in wk 7 . These results provide alternatives to antibiotics for treatment of DD lesions and highlight the potential importance of cleaning feet in the milking parlor.

Key words: topical treatment, intervention, control, lameness, digital dermatitis

\section{INTRODUCTION}

Digital dermatitis (DD) is an infectious bacterial disease affecting the feet of cattle. The disease results in lesions that can cause lameness, production losses, poor fertility, and decreased animal welfare in dairy cattle (Holzhauer et al., 2008). It is considered endemic in dairy farms in much of the world (van Amstel et al., 1995; Rodriguez-Lainz et al., 1998; Wells et al., 1999; Holzhauer et al., 2006; van Andel et al., 2012; Solano et al., 2016), affecting up to $92 \%$ of farms in Europe and North America, with herd-level prevalence ranging from 0 to $74 \%$ (Somers et al., 2005; Holzhauer et al., 2006; Cramer et al., 2008; Solano et al., 2016). The infectious nature of $\mathrm{DD}$, attributed to a complex polybacterial community consistently including multiple Treponema spp. (Gomez et al., 2012; Krull et al., 2014, 2016a), results in spread of lesions after an infected cow is introduced to the herd and is extremely difficult to eradicate once present (Orsel et al., 2017).

Currently, DD detection in the trimming chute is the gold standard, although more efficient detection methods have been developed for use in the milking parlor and during pen walks (Relun et al., 2011; Stokes et al., 2012; Winders et al., 2015; Jacobs et al., 2017; Solano et al., 2017a). In addition, numerous scoring systems have been developed to distinguish disease stages, with the M-stage scoring system (Berry et al., 2012; Döpfer et al., 1997) being the most common and differentiating between active and healing or chronic lesion stages.

On-farm DD control includes prevention and treatment. Preventative practices include herd-level foot bathing (Laven and Logue, 2006) and improved biosecu- 
rity (Wells et al., 1999; Oliveira et al., 2017). Individual cow treatment is typically done during routine hoof trimming, with topical products (often tetracycline or other antimicrobials; Potterton et al., 2012) applied to DD lesions on an infrequent basis. Recommendations for early detection and treatment of DD lesions have been made to improve treatment outcomes and reduce prevalence of DD (Orsel et al., 2017).

The use of tetracycline and oxytetracycline for treating DD lesions in North America is currently off label, has been associated with antibiotic residues in milk (Cramer and Johnson, 2015), and contributes to growing concerns regarding antimicrobial resistance (Tang et al., 2017). Aerosolized oxytetracycline is currently licensed for use in the United Kingdom, and oxytetracycline solution was more efficacious in treating DD compared with a negative control (Britt et al., 1996). Also, Hernandez et al. (1999) reported that oxytetracycline solution was more efficacious than $5 \% \mathrm{CuSO}_{4}$ solution, acidified $\mathrm{CuSO}_{4}$ solution, hydrogen peroxide-peroxyacetic acid, and tap water, with a bacterial spectrum similar to tetracycline and chlortetracycline products. Various treatment regimens for tetracyclines have been tested, resulting in clinical cure rates ranging from 22 to $73 \%$ over 3 to 30 d (Nishikawa and Taguchi, 2008; Berry et al., 2010; Cutler et al., 2013). Additionally, antibiotic products remain difficult to use routinely and difficult to apply because they require animal restraint or application in the milking parlor, where antibiotics should be avoided. Consequently, nonantibiotic topical applications are available commercially and marketed for control of DD; however, few have been clinically tested for effectiveness (Shearer and Hernandez, 2000; Moore et al., 2001). Intra Hoofsol liquid (Diamond Hoof Care Ltd., Intracare BV, Veghel, the Netherlands) and HealMax (AgroChem Inc., Saratoga Spings, NY) are 2 widely used commercially available topical applications marketed for control of DD in dairy cattle. HoofSol is similar to a topical product previously studied (Intra Hoof Fit gel; Intracare BV) and is reported to be more effective at curing ulcerative (M2) lesions compared with chlortetracycline (Holzhauer et al., 2011); however, its efficacy on early lesions and preventing active DD lesions apparently has not been reported. HealMax is a glutaraldehyde-based product, and glutaraldehyde has been reported to be equally effective at killing Treponema bacteria at 30-s and 10-min exposure times with a minimum bactericidal concentration of $0.188 \%$ (vol/vol; Hartshorn et al., 2013). However, randomized clinical trials testing the efficacy of HealMax on DD lesions apparently have not been reported. There is a need to determine effective treatment strategies to routinely identify and promptly treat DD lesions to improve management of DD, decrease prevalence of DD on farms, and improve animal welfare and production. Because early identification and treatment are commonly suggested, the efficacy of topical products to treat DD lesions in early DD lesion stages should be included as opposed to treatment of only ulcerative lesion stages. Also, producers may not differentiate Mstages and may simply treat any visual presentation of DD. Therefore, the objective of this study was to evaluate an 8-wk routine treatment program of HoofSol and HealMax for their ability to (1) transition active DD lesions to nonactive lesions ("clinical cure") and (2) prevent recurrence of active DD lesions compared with positive (tetracycline solution) and negative (saline) controls.

\section{MATERIALS AND METHODS}

\section{Farm and Cow Selection}

A randomized blinded controlled intervention trial was conducted on 10 dairy farms in Alberta, Canada, to evaluate routine use of various topical applications on DD lesions in the milking parlor. Participating farms had (1) a lactating herd prevalence (all stages) of at least $25 \%$ as determined by Jacobs et al. (2017) to achieve an average herd-level prevalence of $\geq 40 \%$ (per the sample size calculation) and (2) cows being milked in a milking parlor, allowing for DD lesion assessment and topical treatment application. Data were collected between January 25 and March 29, 2016. All methods were approved by the University of Calgary Veterinary Sciences Animal Care Committee (AC15-0099).

\section{Sample Size Justification}

To detect a $20 \%$ difference in proportion of active lesions (M1, M2, M4.1) that transitioned to nonactive stages (i.e., clinical cure) in treatment groups compared with the negative control (assuming active treatment will result in $42.9 \%$ of treated lesions healing in $1 \mathrm{wk}$ (Cutler et al., 2013), with an $\alpha$ of 0.013 (Bonferroni correction for 4 comparisons with an $\alpha$ of 0.05 results) and $80 \%$ power, 140 active lesions would be required per treatment group (560 total). Using an average herd size of 200 lactating cows and an average active baseline herd-level DD prevalence of $20 \%, 40$ cows with lesions needed to be recruited per farm in the first week to be assigned weekly to treatment groups. Ten farms would contribute approximately 640 cows with lesions (40 cows with lesions per farm in wk $1 \times 10$ farms $=400)$ in the first week to be identified and treated according to protocol. With an estimated $2 \%$ incidence 
of active lesions per farm per week, an additional 240 active lesions would be assigned to treatment (herd size of $200 \times 2 \%$ incidence $\times 7 \mathrm{wk} \times 10$ farms $=280)$ for a total of 680 active lesions recruited over the course of the study.

\section{Assessment of $D D$}

Digital Dermatitis. Milking parlor DD assessment was performed once weekly for 8 wk according to the protocol described by Solano et al. (2017a). In short, cows' hind feet were cleaned by spraying water from the parlor hose. Thereafter, a mirror (glued to a kitchen spatula) and a headlamp (Tikka Plus 2, Petzl Distribution, Crolles, France) were used to inspect feet. Feet were scored according to the M-stage system (Döpfer et al., 1997; Berry et al., 2012) in which feet were scored as M0 if they had normal skin and no sign of existing lesions, M1 (active) if an epithelial defect $<2 \mathrm{~cm}$ existed, M2 (active) if an active ulcerative lesion $\geq 2$ $\mathrm{cm}$ was present, M3 (healing) if the lesion was covered by brown-black scab-like tissue, M4 (chronic) if hyperkeratotic or proliferative growth was present, and M4.1 (active) if an active lesion $<2 \mathrm{~cm}$ was detected between the borders of an M4 lesion.

Training. Training of observers was completed according to the protocol described by Solano et al. (2017b). Three observers were trained by the first author to identify DD lesion stages using photographs and live evaluations in the milking parlor. Interobserver agreement for identifying DD lesion stages was estimated based on DD scores given by the 3 observers on 40 DD images.

\section{In-Parlor Application}

Randomization. Stratified randomization was used to assign cows identified with any of the DD lesion stages M1, M2, M3, M4, and M4.1 to 1 of the 4 intervention groups. Cows were stratified by herd, and for each farm a randomization list was created in Microsoft Excel (Microsoft Corp., Redmond, WA). In the first visit, cows identified with any DD lesion in 1 or both hind feet were assigned to the next intervention number on the list. Cows identified in subsequent visits that had been previously assigned to an intervention remained in that intervention group throughout the 8-wk trial.

Topical Applications. Four topical applications were evaluated: (1) HoofSol (Diamond Hoof Care Ltd., Intracare BV, Veghel, the Netherlands), (2) HealMax (AgroChem Inc., Saratoga Springs, NY), (3) tetracycline solution (positive control), and (4) $0.9 \%$ saline (negative control; B. Braun Medical Inc., Irvine, CA).
Details on ingredients and manufacturer's usage directions are included in the Appendix. In short, HoofSol contains copper, zinc, and aloe vera, with instructions to apply weekly as a $50 \%$ solution. HealMax is a proprietary blend containing 1.5 pentanedial, alcohol ethoxylate, propylene glycol, xantham gum, tocopherol (vitamin E), methyl salicylate, and water, with instructions to apply 3 to $5 \mathrm{~mL}$ once or twice on the same or consecutive days. Tetracycline powder (tetracycline 250, Vetoquinol Inc., Lavaltrie, QC, Canada) was mixed with $0.9 \%$ saline to achieve an application of 2 to $3 \mathrm{~g}$ of tetracycline (Cutler et al., 2013), and the powder was dissolved completely in solution, with no settling observed. Green food coloring was added to $0.9 \%$ saline (negative control) to assist in blinding researchers and farmers to the applications, as all applications had a yellow-green color. Applications were prepared weekly according to the manufacturers' recommendations (for HealMax and HoofSol) or trial protocol (for tetracycline and saline) before farm visits.

Each solution was placed in a 500-mL opaque spray bottle identified with a code $(\mathrm{A}-\mathrm{D})$ to blind researchers and farmers to treatments. This code was used throughout data collection and analysis. During farm visits, approximately $5 \mathrm{~mL}$ of topical solution was applied to the cleaned lesion site of recruited cattle that had been randomized to each specific treatment. This volume was based on the recommended 3- to 5-mL application for HealMax, which was then used for the other products (in the absence of amount recommendations) to maintain consistency and blinding.

Farmers continued their usual DD management, including footbaths and hoof trimming treatments, throughout the study; however, footbaths were not used during the milking when treatments occurred. Individual animal treatment for DD was recorded, and these cattle were removed from analyses.

Application and Follow-Up. Weekly parlor inspections were performed by the research team during milking at each of the 10 farms for the 8-wk duration of the study. During each parlor inspection, 1 researcher (the scorer) scored the DD status of the hind feet of each cow, and any foot with an M-lesion was marked using stock marker on the hind leg. A second researcher (the treater) randomized each cow according to a computer-generated randomization list. If a cow had a M1 to M4.1 lesion on only 1 hind leg, only the affected leg received the topical application. On subsequent weeks, the treater identified cows previously assigned to an intervention and applied the topical product previously assigned. Consequently, all feet with a DD lesion stage M1 to M4.1 were consistently treated with the same application throughout the study. In the case of M1 
to M4.1 lesions transitioning to M0, the foot was not treated as long as it remained M0; however, if a recurring lesion occurred (M1-M4.1), the foot was retreated with the previously assigned product.

\section{Statistical Analyses}

Data were entered into Microsoft Excel, and statistical analyses were performed using Stata 13.1 (StataCorp, College Station, TX). For all analyses, $P<0.05$ was considered significant. The foot within a cow was considered the statistical unit.

Interobserver agreement was calculated based on comparing the scores of each of the 3 observers during a classroom session with photographs of DD after training was complete. Agreement among observers was calculated as number of exact agreements/total number of observations $\times 100$, whereas actual agreement beyond chance was assessed by kappa ( $\kappa$; Cohen, 1960). Linear weighted kappa $(\kappa \mathbf{w})$ for ordinal scores was calculated to quantify the magnitude of discrepancy among observers using a weight matrix (Cohen, 1968; Fleiss et al., 2003) according to the order M2 > M4.1 > M1 > M4 $>$ M3 > M0 (5 M-stages) and active > chronic $>$ no lesion (simplified scoring system). Agreement as measured using $\kappa$ was interpreted according to Landis and Koch (1977) as $\leq 0=$ poor, 0.01 to $0.20=$ slight, 0.21 to $0.40=$ fair, 0.41 to $0.60=$ moderate, 0.61 to $0.80=$ substantial, and 0.81 to $1.00=$ almost perfect.

All descriptive analyses were performed using the simplified scoring system-no lesions (M0), active lesions (M1, M2, M4.1), and chronic lesions (M3, M4) - based on the stage's infectiousness and clinical relevance (Relun et al., 2011). Additionally, if $>1$ DD lesion was observed on the same foot, the most severe
M-stage was used according to the proposed hierarchy: M2 > M4.1 > M1 > M4 > M3 (Relun et al., 2012; Solano et al., 2017a).

Generalized linear mixed models were used to model mean hazard rates for each outcome, conditional on herd and cow. The logit link was used for all analyses, and time was treated as discrete (Cox, 1972). Outcomes of interest were clinical cure and recurrence of active DD lesions. Clinical cure was defined as transition of active DD (M1, M2, and M4.1) lesions to nonactive stages (M0, M3, and M4). Recurrence was defined as active lesions recurring from nonactive lesions. Clinical cure and recurrence outcome estimates were obtained from the model output and converted to proportions representing the risk of clinical cure or recurrence. Analyses were done at the foot level and considered only the first outcome (clinical cure or recurrence) of all feet within cows at risk that were enrolled at wk 0 and followed throughout the study. Univariable analyses were performed to assess associations between outcomes and covariates and to select variables to include in the final analysis using a manual backward stepwise selection. Farm and cow were included in the final models as random effects. Cow-level variables significant at $P \leq$ 0.05 were retained in the final model for each outcome. Additionally, if confounding was present (i.e., removal of any variable resulted in a $30 \%$ change in the estimate of any other significant predictor), that variable was also retained in the final model. Predictor variables considered included treatment, week of trial, parity, and DIM. Treatment by week was forced into the final models to account for time and present varying hazards over time. Two-way biologically relevant interactions (e.g., parity and DIM, parity and treatment, and DIM and treatment) were tested among significant predic-

Table 1. Farm characteristics (mean $\pm \mathrm{SD}$ ) and digital dermatitis (DD) prevalence for 10 freestall dairy farms in Alberta, Canada

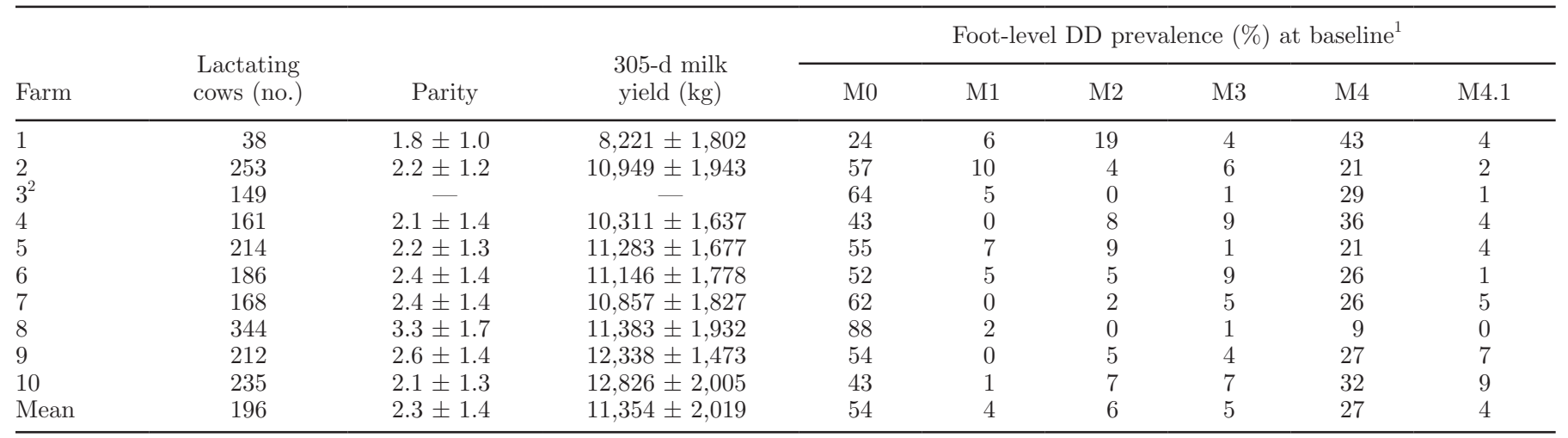

${ }^{1}$ Apparent prevalence detected in milking parlor. M0 = normal skin and no sign of existing lesions; M1 (active) = epithelial defect $<2 \mathrm{~cm}$ present: M2 (active) = active ulcerative lesion $\geq 2 \mathrm{~cm}$ present; M3 (healing) = lesion covered by brown-black scab-like tissue; M4 (chronic) = hyperkeratotic or proliferative growth present; M4.1 (active) = active lesion $<2 \mathrm{~cm}$ detected between the borders of an M4 lesion.

${ }^{2}$ Not enrolled in DHI; parity and 305-d milk yield excluded from overall mean. 
tors in the main effects model, but none were retained $(P>0.05$ in all cases $)$.

\section{RESULTS}

\section{Farms}

Average herd size was 196 lactating cows (range: $38-344$ ) with a mean $305-d$ milk yield of $11,354 \mathrm{~kg}$ and mean parity of 2.3 (Table 1). At baseline (wk 0), mean apparent within-herd foot-level DD prevalence was $46 \%$ (range: $12-76 \%$ ) and prevalence of feet with active lesions was 14\% (range: 3-28\%). Furthermore, $21 \%$ of cows scored at baseline had DD lesions on only 1 foot, whereas $32 \%$ had DD lesions on both hind feet.

A total of 32,664 observations on 2,475 lactating cows were collected over the 8 -wk study interval (Figure 1). Of the 2,475 cows enrolled, 1,138 cows did not have any DD lesions (M1-M4.1) at wk 0, 74 cows were excluded due to treatment or recording errors, and 193 cows were excluded due to being treated by the farmer or hoof trimmer, resulting in 15,600 observations on 1,070 cows (1,372 feet) available for the final analyses.

Despite randomization, after exclusions more cows were enrolled in the HealMax group (27\%) followed by the tetracycline group (26\%), and equal proportions of

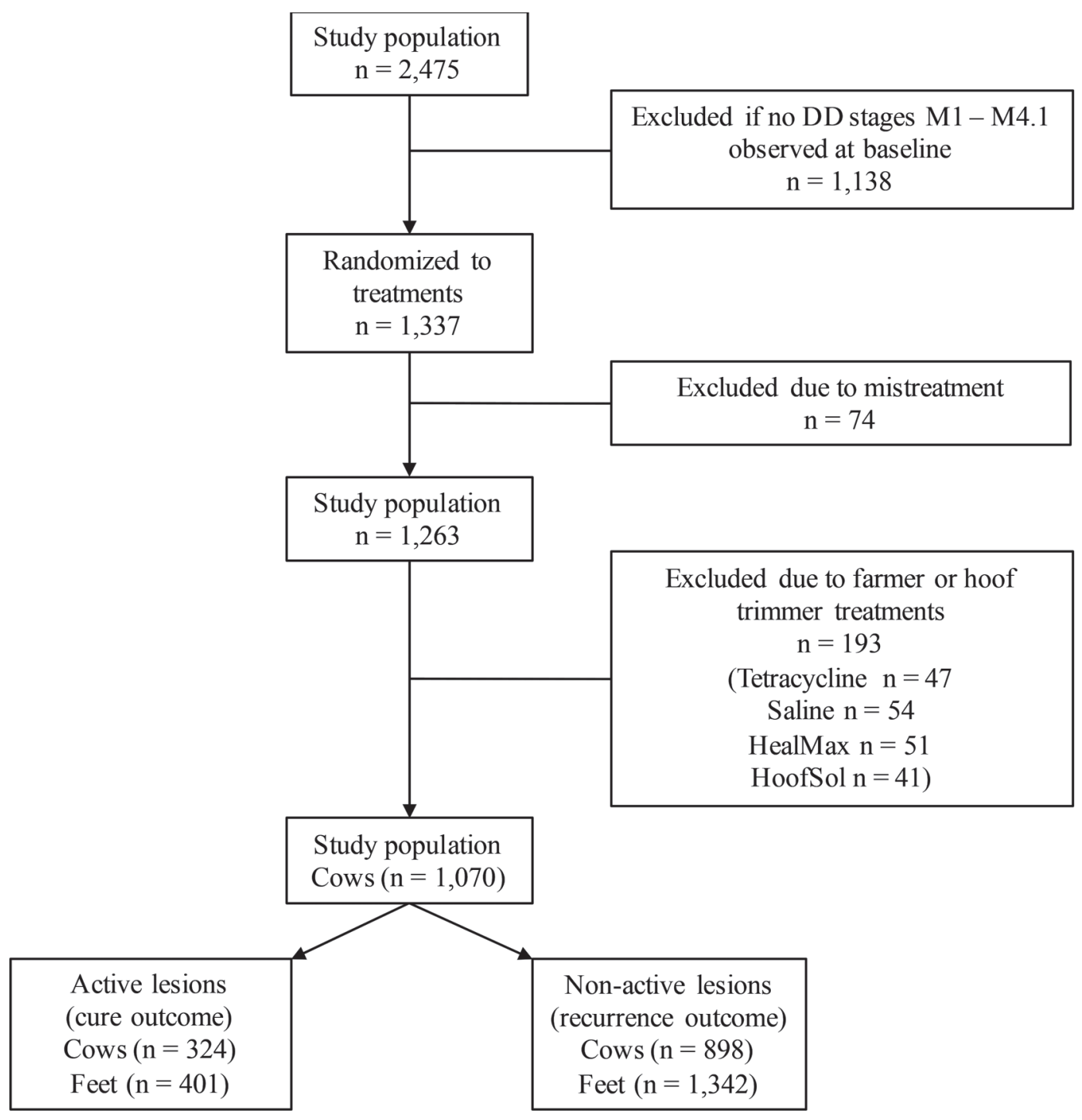

Figure 1. Enrollment flow chart. Tetracycline manufactured by Vetoquinol Inc. (Lavaltrie, QC, Canada); HealMax manufactured by AgroChem Inc. (Saratoga Springs, NY); HoofSol manufactured by Diamond Hoof Care Ltd. (Intracare BV, Veghel, the Netherlands). DD = digital dermatitis. $\mathrm{M} 0=$ normal skin and no sign of existing lesions; $\mathrm{M} 1$ (active) $=$ epithelial defect $<2 \mathrm{~cm}$ present; M2 (active) = active ulcerative lesion $\geq 2 \mathrm{~cm}$ present; M3 (healing) = lesion covered by brown-black scab-like tissue; M4 (chronic) = hyperkeratotic or proliferative growth present; M4.1 (active) = active lesion $<2 \mathrm{~cm}$ detected between the borders of an M4 lesion. 
cows were enrolled in the saline $(23 \%)$ and HoofSol (24\%) groups (Table 2). Fewer cows in the HoofSol group (21\%) were excluded due to farmer or hoof trimmer treatments compared with the saline group $(P=$ 0.03 ), and an equivalent number of farmer or hoof trimmer treatments occurred among the tetracycline, HealMax, and saline groups $(24,26$, and $28 \%$, respectively).

\section{Interobserver Agreement}

Interobserver agreement for scoring DD in the milking parlor was substantial in all cases, with agreement $\geq 80 \%, \kappa=0.75(95 \%$ CI: $0.65-0.82)$, and $\kappa_{\mathrm{w}}=0.75$ (95\% CI: $0.73-0.85)$ for the simplified scoring between the 3 observers. Applying $\kappa_{\mathrm{w}}$ had little to no effect for the simplified scoring system.

\section{Clinical Cure}

A total of 324 cows and 401 feet with active lesions were used in the analysis for clinical cure (Figure 1). Of the 401 feet with active lesions in wk 1, 393 (91\%) were cured clinically within the 8 -wk study period. The mean number of treatments until clinical cure occurred was $3,2,3$, and 3 for saline, tetracycline, HealMax, and HoofSol, respectively. The probability of clinical cure for active lesions in wk 1 was 34, 69, 52, and $79 \%$ for saline, tetracycline, HealMax, and HoofSol, respectively (Tables 3 and 4). Compared with the wk 1 probability of clinical cure with saline, tetracycline, HealMax, and HoofSol were more effective at promoting clinical cure, but only in the first week (Table 3 ). In wk 2 , the probability of clinical cure of active lesions was 35, 70, 43, and $51 \%$ for saline, tetracycline, HealMax, and HoofSol, respectively. After 8 wk, the probability of clinical cure for saline, tetracycline, HealMax, and HoofSol was 10 , 33,31 , and $45 \%$, with no difference among treatments.

\section{Recurrence}

A total of 898 cows and 1,342 feet were used in the analysis for recurrence of active lesions from nonactive DD stages. Of the 1,342 feet with nonactive lesions, $590(43 \%)$ recurred within the 8 -wk study period. The mean number of treatments until recurrence was 4 for each treatment group. The probability of recurrence of active lesions in wk 1 was $9,11,11$, and $8 \%$ for saline, tetracycline, HealMax, and HoofSol, respectively (Tables 5 and 6 ). Compared with the wk 1 probability of recurrence with saline, no product was more effective at preventing recurrence (Tables 5 and 6 ). In wk 2, the probability of recurrence with tetracycline, HealMax, and HoofSol was equivalent to saline, with probability of recurrence being $7,12,7$, and $5 \%$ for saline, tetracycline, HealMax, and HoofSol, respectively. After 8 wk, the probability of recurrence with saline, tetracycline, HealMax, and HoofSol was 5, 7, 6, and 6\%, with no difference among treatments. For all analyses, neither DIM nor parity modified the effect of treatment on either outcome.

\section{DISCUSSION}

The commercially available topical treatments HealMax and HoofSol were as effective as tetracycline and more effective than saline at clinically curing active lesions with 1 treatment. However, over an 8-wk treatment routine, there was no difference among applications for clinically curing or preventing active DD lesions. Hosing feet and applying saline resulted in a

Table 2. Distribution (\%) of cows and lesions randomized to each treatment at wk 0 ( $\mathrm{n}=1,070$ cows; 1,372 feet)

\begin{tabular}{lccccc}
\hline & \multicolumn{5}{c}{ Topical product $^{2}$} \\
\cline { 2 - 6 } & \multicolumn{1}{c}{$\begin{array}{c}\text { Negative } \\
\text { control }\end{array}$} & Tetracycline & HealMax & HoofSol & Total \\
\hline Item & $251(23)$ & $279(26)$ & $287(27)$ & $253(24)$ & 1,070 \\
Fows & $317(23)$ & $375(27)$ & $353(26)$ & $327(24)$ & 1,372 \\
Active DD & $76(19)$ & $105(26)$ & $125(31)$ & $100(25)$ & 406 \\
Chronic DD & $241(25)$ & $270(28)$ & $228(24)$ & $227(23)$ & 966 \\
\hline
\end{tabular}

${ }^{1}$ Active DD lesions include M1, M2, and M4.1 according to Döpfer et al. (1997) and Berry et al. (2012). Chronic DD lesions include M3 and M4 according to Döpfer et al. (1997). M0 = normal skin and no sign of existing lesions; M1 (active) = epithelial defect $<2 \mathrm{~cm}$ present; M2 (active) = active ulcerative lesion $\geq 2 \mathrm{~cm}$ present; M3 (healing) = lesion covered by brown-black scab-like tissue; M4 (chronic) = hyperkeratotic or proliferative growth present; M4.1 (active) = active lesion $<2 \mathrm{~cm}$ detected between the borders of an M4 lesion.

${ }^{2}$ Tetracycline manufactured by Vetoquinol Inc. (Lavaltrie, QC, Canada); HealMax manufactured by AgroChem Inc. (Saratoga Springs, NY); HoofSol manufactured by Diamond Hoof Care Ltd., Intracare BV (Veghel, the Netherlands). 
probability of clinical cure of $33 \%$ in wk 1, which may highlight the potential importance of maintaining foot hygiene by hosing feet in the milking parlor or may be due to a healing effect of saline.

The clinical cure rate, defined as transitioning from active to nonactive stages, was used as an outcome as opposed to "healing," as the latter implies that a foot would return to an unaffected state (M0), which is unlikely in 7 wk (Krull et al., 2016b). In that regard, the goal of DD control is to achieve a manageable state of disease through limiting the prevalence of active lesions (Döpfer, 2009). Consequently, transition and recurrence should be considered the most relevant in terms of on-farm DD control.
Tetracycline and oxytetracycline are commonly used as a treatment for DD by farmers, veterinarians, and hoof trimmers (Laven and Logue, 2006; Potterton et al., 2012) and are often used as a positive control in DD treatment studies using various application methods with varying results. This heterogeneity in treatment regimens has resulted in clinical cure rates ranging from 22 to $73 \%$ over 3 to 30 d (Nishikawa and Taguchi, 2008; Berry et al., 2010; Cutler et al., 2013). Topical treatment with tetracycline in our study was better only in the first week after the first treatment compared with the negative control (saline). This could be due to the application method, which may have resulted in only limited contact of the product to the lesion (compared

Table 3. Final generalized linear mixed model of the clinical cure of active (M1, M2, M4.1) digital dermatitis lesion stages to nonactive (M0, M3, M4) stages for 4 topical treatments over 8 wk $^{1}$ ( $\mathrm{n}=324$ cows; 401 feet)

\begin{tabular}{|c|c|c|c|c|c|}
\hline Item & $\begin{array}{l}\text { Coefficient } \\
(\beta)\end{array}$ & SE & $\begin{array}{l}\text { Hazard } \\
\text { ratio }\end{array}$ & $P$-value & $\begin{array}{c}95 \% \text { CI } \\
\text { of hazard ratio }\end{array}$ \\
\hline Intercept & -0.68 & 0.36 & - & 0.06 & - \\
\hline \multicolumn{6}{|l|}{ Treatment $^{2}$} \\
\hline Saline & Referent & - & - & - & - \\
\hline Tetracycline & 1.48 & 0.43 & 4.37 & $<0.01$ & $1.89-10.13$ \\
\hline HealMax & 0.77 & 0.40 & 2.17 & 0.05 & $0.99-4.75$ \\
\hline HoofSol & 2.01 & 0.46 & 7.47 & $<0.01$ & $3.01-18.49$ \\
\hline \multicolumn{6}{|l|}{ Week } \\
\hline 1 & Referent & - & - & - & - \\
\hline 2 & 0.08 & 0.47 & 1.09 & 0.86 & $0.43-2.75$ \\
\hline 3 & -0.03 & 0.59 & 0.97 & 0.96 & $0.30-3.09$ \\
\hline 4 & 0.28 & 0.63 & 1.32 & 0.66 & $0.39-4.50$ \\
\hline 5 & -0.69 & 0.89 & 0.50 & 0.44 & $0.09-2.88$ \\
\hline 6 & 1.10 & 0.74 & 3.00 & 0.14 & $0.70-12.84$ \\
\hline 7 & -1.52 & 1.40 & 0.22 & 0.28 & $0.01-3.42$ \\
\hline \multicolumn{6}{|c|}{ Treatment by week } \\
\hline Saline & Referent & - & - & - & - \\
\hline \multicolumn{6}{|l|}{ Tetracycline } \\
\hline Wk 2 & -0.02 & 0.64 & 0.98 & 0.98 & $0.28-3.47$ \\
\hline Wk 3 & 0.18 & 0.85 & 1.20 & 0.83 & $0.22-6.38$ \\
\hline Wk 4 & -0.72 & 1.00 & 0.49 & 0.47 & $0.07-3.44$ \\
\hline Wk 5 & 0 & - & - & - & - \\
\hline Wk 6 & -2.40 & 1.34 & 0.09 & 0.07 & $0.01-1.25$ \\
\hline Wk 7 & 0 & - & - & - & - \\
\hline \multicolumn{6}{|l|}{ HealMax } \\
\hline Wk 2 & -0.45 & 0.59 & 0.64 & 0.45 & $0.20-2.04$ \\
\hline Wk 3 & -0.07 & 0.71 & 0.93 & 0.92 & $0.23-3.72$ \\
\hline Wk 4 & -0.27 & 0.76 & 0.76 & 0.73 & $0.17-3.42$ \\
\hline Wk 5 & 0.72 & 1.05 & 2.06 & 0.49 & $0.26-16.19$ \\
\hline Wk 6 & -0.78 & 0.95 & 0.46 & 0.41 & $0.07-2.96$ \\
\hline Wk 7 & 0.62 & 1.78 & 1.85 & 0.73 & $0.06-60.75$ \\
\hline \multicolumn{6}{|l|}{ HoofSol } \\
\hline Wk 2 & -1.38 & 0.70 & 0.25 & 0.05 & $0.06-0.99$ \\
\hline Wk 3 & 0.08 & 0.86 & 1.08 & 0.93 & $0.20-5.89$ \\
\hline Wk 4 & -1.23 & 1.00 & 0.29 & 0.22 & $0.04-2.08$ \\
\hline Wk 5 & -0.09 & 1.27 & 0.92 & 0.95 & $0.08-11.07$ \\
\hline Wk 6 & -1.74 & 1.14 & 0.18 & 0.13 & $0.02-1.65$ \\
\hline Wk 7 & 0 & - & - & - & - \\
\hline
\end{tabular}

${ }^{1} \mathrm{M} 0=$ normal skin and no sign of existing lesions; M1 (active) = epithelial defect $<2 \mathrm{~cm}$ present; M2 (active) = active ulcerative lesion $\geq 2 \mathrm{~cm}$ present; M3 (healing) = lesion covered by brown-black scab-like tissue; M4 (chronic) = hyperkeratotic or proliferative growth present; M4.1 (active) = active lesion $<2 \mathrm{~cm}$ detected between the borders of an M4 lesion.

${ }^{2}$ Tetracycline manufactured by Vetoquinol Inc. (Lavaltrie, QC, Canada); HealMax manufactured by AgroChem Inc. (Saratoga Springs, NY); HoofSol manufactured by Diamond Hoof Care Ltd., Intracare BV (Veghel, the Netherlands). 
with tetracycline powder and a wrap), or perhaps the difference between the negative control and tetracycline was not significantly different after wk 1 due to the high probability of clinical cure of the negative control.

HoofSol is a copper- and zinc-based product; a comparable product, Intra Hoof Fit gel (Intracare BV), has been studied as a treatment compared with chlortetracycline spray (Eurovet Animal Health, Bladel, the Netherlands; (Holzhauer et al., 2011). This Dutch study, which included 205 hind feet with M2 lesions from 172 cows from 5 farms, reported that 3 treatments with Intra Hoof Fit gel on d 0, 3, and 7 were more effective at curing M2 lesions (cure was defined as transition to M0 or M4) compared with 3 treatments with chlortetracycline spray on $\mathrm{d} 0,1$, and 2 after d 28. The higher clinical cure rate in the Dutch study may have been due to the application method. In that regard, Intra Hoof Fit gel was painted on the lesion in the hoof trimming chute and covered with a bandage, as opposed to the topical spray application used in this study. Although both have merit, the ease of application using a spray bottle in the parlor was more conducive to routine treatment.

No previous reports exist of the effect of HealMax on digital dermatitis lesions; however, Manske et al. (2002) compared the use of $5 \mathrm{~mL}$ of another glutaraldehyde product (Parvocide, Coventry Chemical, Coventry, UK) with the use of $5 \mathrm{~mL}$ of oxytetracycline solution at a concentration of $100 \mathrm{mg} / \mathrm{mL}$ on DD lesions. Treatments were applied with bandages and treatment was repeated after $5 \mathrm{~d}$; bandages were removed $2 \mathrm{~d}$ after the second treatment. Glutaraldehyde was less effective compared with oxytetracycline solution and equivalent to hoof trimming alone. These results differed from the current study, perhaps due to the formula of the product or the application method (in the hoof trimming chute with a bandage compared with spray in the milking parlor).

Additionally, grouping all active lesions (M1, M2, M4.1) in the current study may have diluted the effect of treatment if a less prevalent stage was more susceptible compared with a more prevalent stage. Future studies should focus on treatment of specific M-stages to determine which lesions are most susceptible so that treatment can be targeted to improve outcomes (Orsel et al., 2017).

Similar to this study, Cutler et al. (2013) reported equivalence in recurrence between treatments with tetracycline paste and powder applied with a bandage and speculated that topical applications were potentially limited in reaching the location of infection to prevent recurrence (Mumba et al., 1999; Nordhoff et al., 2008). Additionally, Krull et al. (2016b) determined the average time for lesion development in the absence of treatment and prevention procedures to be $133 \mathrm{~d}$. Although the time frame of the current study did not allow for a long follow-up to study continued recurrence of active lesions, it is noteworthy that the weekly probability of recurrence for all treatments did not exceed $12 \%$ throughout the study.

In our study, multiple products were randomized at the cow level on multiple farms to improve extrapolation of results and external validity (Relun et al., 2012). Although randomization did not result in equal numbers of lesions per treatment group, proper randomization resulting in unequal groups may be preferable to forcing equality among treatment groups (Schulz and Grimes, 2002). Additionally, weekly inspections limited

Table 4. Probability (95\% CI in parentheses) of clinical cure based on the final generalized linear mixed model of the clinical cure of active (M1, M2, M4.1) digital dermatitis lesion stages to nonactive (M0, M3, M4) stages for 4 topical treatments over $8 \mathrm{wk}^{1}(\mathrm{n}=324$ cows; 401 feet $)$

\begin{tabular}{lcccc}
\hline & \multicolumn{4}{c}{ Treatment $^{2}$} \\
\cline { 2 - 5 } Week & Saline & Tetracycline & HealMax & HoofSol \\
\hline 1 & $33.5(19.8-50.7)$ & $68.8(53.5-80.8)$ & $52.2(37.7-66.4)$ & $79.0(64.5-88.6)$ \\
2 & $35.4(18.8-56.5)$ & $70.2(45.4-87.0)$ & $43.2(25.0-63.4)$ & $50.6(24.3-76.5)$ \\
3 & $32.8(14.0-59.4)$ & $71.9(36.9-91.8)$ & $49.7(26.7-72.8)$ & $79.8(46.6-94.7)$ \\
4 & $40.0(17.3-68.0)$ & $58.7(18.9-89.7)$ & $52.4(25.8-77.8)$ & $59.1(19.0-89.9)$ \\
5 & $20.2(4.4-58.0)$ & $52.5(14.1-88.2)$ & $53.0(21.1-82.6)$ & $63.3(17.3-93.4)$ \\
6 & $60.2(27.5-85.7)$ & $37.6(4.8-87.8)$ & $60.0(22.9-88.3)$ & $66.5(20.6-93.8)$ \\
7 & $10.0(0.7-65.0)$ & $32.6(2.7-89.4)$ & $30.8(3.9-83.0)$ & $45.3(4.8-93.2)$ \\
\hline
\end{tabular}

${ }^{1} \mathrm{M} 0=$ normal skin and no sign of existing lesions; M1 (active) $=$ epithelial defect $<2 \mathrm{~cm}$ present; M2 (active) $=$ active ulcerative lesion $\geq 2 \mathrm{~cm}$ present; M3 (healing) = lesion covered by brown-black scab-like tissue; M4 (chronic) = hyperkeratotic or proliferative growth present; M4.1 (active) $=$ active lesion $<2 \mathrm{~cm}$ detected between the borders of an M4 lesion.

${ }^{2}$ Tetracycline manufactured by Vetoquinol Inc. (Lavaltrie, QC, Canada); HealMax manufactured by AgroChem Inc. (Saratoga Springs, NY); HoofSol manufactured by Diamond Hoof Care Ltd., Intracare BV (Veghel, the Netherlands). 
the probability of missing lesion transitions, although early stages may transition more rapidly (Nielsen et al., 2012). Blinding of the research team and farmers ensured that compliance was maintained and limited the bias of outcome assessments.

It is noteworthy that washing feet with running water and applying saline resulted in a relatively high clinical cure rate. If product application seems too cumbersome to include as part of a DD control program, hosing the feet in the parlor (which would need to be done to apply product) may assist in promoting clinical cure of active lesion stages. In fact, Thomsen et al. (2012) reported that feet washed with water and $0.4 \%$ soap were less likely to have DD compared with feet left unwashed. When only water was used, the positive effect of washing was no longer significant. However, no footbaths were used in the Thomsen study, and washing feet before footbathing may improve contact with the footbath solution (Watson, 2007) and reduce contamination (Manning et al., 2017). Unfortunately, as clinical cure rates of tetracycline, HealMax, and HoofSol appeared to be equivalent to the negative after wk 2 , we are unable to determine whether this was due to hosing the feet in the parlor or natural lesion improvement,

Table 5. Final generalized linear mixed model of the recurrence of active (M1, M2, M4.1) digital dermatitis lesion stages from chronic (M3, M4) stages for each of 4 topical treatments over $8 \mathrm{wk}^{1}$ ( $\mathrm{n}=898$ cows; 1,342 feet)

\begin{tabular}{|c|c|c|c|c|c|}
\hline Item & $\begin{array}{l}\text { Coefficient } \\
(\beta)\end{array}$ & $\mathrm{SE}$ & $\begin{array}{l}\text { Hazard } \\
\text { ratio }\end{array}$ & $P$-value & $\begin{array}{c}95 \% \text { CI } \\
\text { of hazard ratio }\end{array}$ \\
\hline Intercept & -2.27 & 0.31 & - & $<0.01$ & - \\
\hline \multicolumn{6}{|l|}{ Treatment $^{2}$} \\
\hline Saline & Referent & - & - & - & - \\
\hline Tetracycline & 0.21 & 0.25 & 1.24 & 0.40 & $0.75-2.03$ \\
\hline HealMax & 0.14 & 0.26 & 1.15 & 0.59 & $0.69-1.90$ \\
\hline HoofSol & -0.23 & 0.27 & 0.80 & 0.41 & $0.47-1.36$ \\
\hline \multicolumn{6}{|l|}{ Week } \\
\hline 1 & Referent & - & - & - & - \\
\hline 2 & -0.35 & 0.28 & 0.71 & 0.21 & $0.41-1.22$ \\
\hline 3 & -0.66 & 0.33 & 0.52 & 0.05 & $0.27-0.99$ \\
\hline 4 & -0.08 & 0.30 & 0.92 & 0.78 & $0.52-1.64$ \\
\hline 5 & 0.29 & 0.30 & 1.34 & 0.33 & $0.75-2.39$ \\
\hline 6 & -0.64 & 0.40 & 0.53 & 0.11 & $0.24-1.16$ \\
\hline 7 & -0.75 & 0.44 & 0.47 & 0.09 & $0.20-1.13$ \\
\hline \multicolumn{6}{|c|}{ Treatment by week } \\
\hline Saline & Referent & - & - & - & - \\
\hline \multicolumn{6}{|l|}{ Tetracycline } \\
\hline Wk 2 & 0.41 & 0.37 & 1.51 & 0.27 & $0.73-3.13$ \\
\hline Wk 3 & 0.36 & 0.44 & 1.43 & 0.42 & $0.61-3.36$ \\
\hline Wk 4 & -0.80 & 0.46 & 0.45 & 0.08 & $0.18-1.11$ \\
\hline Wk 5 & -0.62 & 0.43 & 0.54 & 0.15 & $0.23-1.24$ \\
\hline Wk 6 & 0.35 & 0.52 & 1.42 & 0.50 & $0.52-3.90$ \\
\hline Wk 7 & 0.21 & 0.57 & 1.23 & 0.71 & $0.40-3.80$ \\
\hline \multicolumn{6}{|l|}{ HealMax } \\
\hline Wk 2 & -0.11 & 0.40 & 0.89 & 0.78 & $0.41-1.94$ \\
\hline Wk 3 & 0.39 & 0.43 & 1.48 & 0.37 & $0.63-3.46$ \\
\hline Wk 4 & -0.64 & 0.45 & 0.53 & 0.15 & $0.22-1.27$ \\
\hline Wk 5 & -0.75 & 0.44 & 0.47 & 0.09 & $0.20-1.11$ \\
\hline Wk 6 & 0.26 & 0.52 & 1.30 & 0.62 & $0.47-3.60$ \\
\hline Wk 7 & 0.03 & 0.59 & 1.03 & 0.95 & $0.33-3.26$ \\
\hline \multicolumn{6}{|l|}{ HoofSol } \\
\hline Wk 2 & -0.05 & 0.42 & 0.96 & 0.91 & $0.42-2.16$ \\
\hline Wk 3 & 0.51 & 0.45 & 1.67 & 0.25 & $0.69-4.04$ \\
\hline Wk 4 & -0.11 & 0.43 & 0.90 & 0.81 & $0.38-2.11$ \\
\hline Wk 5 & -0.68 & 0.46 & 0.51 & 0.14 & $0.21-1.26$ \\
\hline Wk 6 & 0.51 & 0.53 & 1.67 & 0.33 & $0.59-4.72$ \\
\hline Wk 7 & 0.53 & 0.57 & 1.70 & 0.35 & $0.55-5.27$ \\
\hline
\end{tabular}

${ }^{1} \mathrm{M} 0=$ normal skin and no sign of existing lesions; M1 (active) = epithelial defect $<2 \mathrm{~cm}$ present; M2 (active) $=$ active ulcerative lesion $\geq 2 \mathrm{~cm}$ present; M3 (healing) = lesion covered by brown-black scab-like tissue; M4 (chronic) $=$ hyperkeratotic or proliferative growth present; M4.1 (active) $=$ active lesion $<2 \mathrm{~cm}$ detected between the borders of an M4 lesion.

${ }^{2}$ Tetracycline manufactured by Vetoquinol Inc. (Lavaltrie, QC, Canada); HealMax manufactured by AgroChem Inc. (Saratoga Springs, NY); HoofSol manufactured by Diamond Hoof Care Ltd., Intracare BV (Veghel, the Netherlands). 
Table 6. Probability (95\% CI in parentheses) of recurrence based on the final generalized linear mixed model of the recurrence of active (M1, M2, M4.1) digital dermatitis lesion stages from chronic (M3, M4) stages for each of 4 topical treatments over $8 \mathrm{wk}^{1}(\mathrm{n}=898$ cows; 1,342 feet $)$

\begin{tabular}{|c|c|c|c|c|}
\hline \multirow[b]{2}{*}{ Week } & \multicolumn{4}{|c|}{ Treatment $^{2}$} \\
\hline & Saline & Tetracycline & HealMax & HoofSol \\
\hline 1 & $9.4(5.4-15.9)$ & $11.4(6.7-18.8)$ & $10.7(6.1-17.8)$ & $7.6(4.2-13.4)$ \\
\hline 2 & $6.8(3.7-12.3)$ & $12.0(7.0-20.0)$ & $7.0(3.7-12.7)$ & $5.3(2.7-10.0)$ \\
\hline 3 & $5.1(2.5-10.0)$ & $8.7(4.6-15.6)$ & $8.3(4.5-15.0)$ & $6.7(3.5-12.4)$ \\
\hline 4 & $8.7(4.7-15.5)$ & $5.0(2.4-10.5)$ & $5.5(2.6-11.0)$ & $6.4(3.3-12.2)$ \\
\hline 5 & $12.2(6.8-21.0)$ & $8.4(4.3-15.9)$ & $7.0(3.5-13.6)$ & $5.3(2.5-10.9)$ \\
\hline 6 & $5.2(2.3-11.2)$ & $8.7(4.3-16.8)$ & $7.5(3.7-14.8)$ & $6.8(3.3-13.4)$ \\
\hline 7 & $4.7(1.9-10.9)$ & $7.0(3.2-14.5)$ & $5.5(2.5-12.0)$ & $6.3(2.9-12.9)$ \\
\hline
\end{tabular}

${ }^{1} \mathrm{M} 0=$ normal skin and no sign of existing lesions; M1 (active) = epithelial defect $<2 \mathrm{~cm}$ present; M2 (active) = active ulcerative lesion $\geq 2 \mathrm{~cm}$ present; M3 (healing) = lesion covered by brown-black scab-like tissue; $\mathrm{M} 4($ chronic) $=$ hyperkeratotic or proliferative growth present; M4.1 (active) = active lesion $<2 \mathrm{~cm}$ detected between the borders of an M4 lesion.

${ }^{2}$ Tetracycline manufactured by Vetoquinol Inc. (Lavaltrie, QC, Canada); HealMax manufactured by AgroChem Inc. (Saratoga Springs, NY); HoofSol manufactured by Diamond Hoof Care Ltd., Intracare BV (Veghel, the Netherlands).

which can occur over the first 7 to $10 \mathrm{~d}$ without any treatment (Blowey and Sharp, 1988).

\section{CONCLUSIONS}

Weekly routine treatment with tetracycline, HealMax, or Hoofsol was no more effective than saline in clinically curing active lesions to nonactive stages in this 8-wk study. Hosing feet in the milking parlor on a weekly basis may improve foot hygiene, improve contact time of the footbath being used, and assist in controlling DD. Furthermore, no product was superior to saline in preventing recurrence of active lesions from nonactive DD stages. These results provided an opportunity to decrease off-label antibiotic use on farm and highlighted the importance of cleaning DD-affected feet (e.g., washing feet with a hose in the milking parlor).

\section{ACKNOWLEDGMENTS}

This study was funded by Alberta Milk (Edmonton, AB, Canada) and Growing Forward 2-Biosecurity Delivery Agent (Edmonton, AB, Canada). The authors thank participating farmers for their willingness, time, and enthusiasm. We are especially grateful for the cooperation of hoof trimmers Elbert Koster (No Tilt Hooftrimming, Spruceview, AB, Canada) and Rob Geier (Casper Hooftrimming, Olds, AB, Canada) and for the contributions of Charlotte Pickel (University of Calgary, Calgary, AB, Canada), Marlieke Pronk (Utrecht University, Utrecht, the Netherlands), Marline van Milne (Utrecht University), and Joanna Wong (University of British Columbia, Vancouver, BC, Canada) to the data collection. We thank John Kastelic (University of Calgary) for editing the manuscript.

\section{REFERENCES}

Berry, S. L., D. H. Read, T. R. Famula, A. Mongini, and D. Döpfer. 2012. Long-term observations on the dynamics of bovine digital dermatitis lesions on a California dairy after topical treatment with lincomycin HCl. Vet. J. 193:654-658.

Berry, S. L., D. H. Read, R. L. Walker, and T. R. Famula. 2010. Clinical, histologic, and bacteriologic findings in dairy cows with digital dermatitis (footwarts) one month after topical treatment with lincomycin hydrochloride or oxytetracycline hydrochloride. J. Am. Vet. Med. Assoc. 237:555-560.

Blowey, R. W., and M. W. Sharp. 1988. Digital dermatitis in dairy cattle. Vet. Rec. 122:505-508.

Britt, J. S., J. Gaska, E. F. Garrett, D. Konkle, and M. Mealy. 1996. Comparison of topical application of three products for treatment of papillomatous digital dermatitis in dairy cattle. J. Am. Vet. Med. Assoc. 209:1134-1136.

Cohen, J. 1960. A coefficient of agreement for nominal scales. Educ. Psychol. Meas. 20:37-46.

Cohen, J. 1968. Weighted kappa: Nominal scale agreement provision for scaled disagreement or partial credit. Psychol. Bull. 70:213-220.

Cox, D. R. 1972. Regression models and life-tables. J. R. Stat. Soc. B. Stat. Methodol. 34:187-220.

Cramer, G., and R. Johnson. 2015. Evaluation of risks of violative milk residues following extra label topical administration of tetracycline for digital dermatitis in dairy cattle. Page 141 in Proc. Intern. Lameness in Ruminants Conf., Valdivia, Chile.

Cramer, G., K. D. Lissemore, C. L. Guard, K. E. Leslie, and D. F. Kelton. 2008. Herd- and cow-level prevalence of foot lesions in Ontario dairy cattle. J. Dairy Sci. 91:3888-3895

Cutler, J. H. H., G. Cramer, J. J. Walter, S. T. Millman, and D. F. Kelton. 2013. Randomized clinical trial of tetracycline hydrochloride bandage and paste treatments for resolution of lesions and pain associated with digital dermatitis in dairy cattle. J. Dairy Sci. 96:7550-7557.

Döpfer, D. 2009. The dynamics of digital dermatitis in dairy cattle and the manageable state of disease. Pages 1-5 in Proc. CanWest Veterinary Conference, Banff, AB, Canada.

Döpfer, D., A. Koopmans, F. A. Meijer, I. Szakall, Y. H. Schukken, W. Klee, R. B. Bosma, J. L. Cornelisse, A. J. van Asten, and A. A. ter Huurne. 1997. Histological and bacteriological evaluation of digital dermatitis in cattle, with special reference to spirochaetes and Campylobacter faecalis. Vet. Rec. 140:620-623.

Fleiss, J. L., B. Levin, and M. C. Paik. 2003. Statistical Methods for Rates and Proportions. J. Wiley and Sons, Hoboken, NJ. 
Gomez, A., N. B. Cook, N. D. Bernardoni, J. Rieman, A. F. Dusick, R. Hartshorn, M. T. Socha, D. H. Read, and D. Döpfer. 2012. An experimental infection model to induce digital dermatitis infection in cattle. J. Dairy Sci. 95:1821-1830.

Hartshorn, R. E., E. C. Thomas, K. Anklam, M. G. Lopez-Benavides, M. Buchalova, T. C. Hemling, and D. Döpfer. 2013. Minimum bactericidal concentration of disinfectants evaluated for bovine digital dermatitis-associated Treponema phagedenis-like spirochetes. J. Dairy Sci. 96:3034-3038.

Hernandez, J., J. K. Shearer, and J. B. Elliott. 1999. Comparison of topical application of oxytetracycline and four nonantibiotic solutions for treatment of papillomatous digital dermatitis in dairy cows. J. Am. Vet. Med. Assoc. 214:688-690.

Holzhauer, M., C. J. Bartels, M. van Barneveld, C. Vulders, and T. Lam. 2011. Curative effect of topical treatment of digital dermatitis with a gel containing activated copper and zinc chelate. Vet. Rec. 169:555.

Holzhauer, M., C. J. M. Bartels, D. Döpfer, and G. van Schaik. 2008. Clinical course of digital dermatitis lesions in an endemically infected herd without preventive herd strategies. Vet. J. 177:222-230.

Holzhauer, M., C. Hardenberg, C. J. M. Bartels, and K. Frankena. 2006. Herd- and cow-level prevalence of digital dermatitis in The Netherlands and associated risk factors. J. Dairy Sci. 89:580-588.

Jacobs, C., K. Orsel, and H. W. Barkema. 2017. Prevalence of digital dermatitis in young stock in Alberta, Canada, using pen walks. J. Dairy Sci. 100:9234-9244.

Krull, A. C., V. L. Cooper, J. W. Coatney, J. K. Shearer, P. J. Gorden, and P. J. Plummer. 2016a. A highly effective protocol for the rapid and consistent induction of digital dermatitis in Holstein calves. PLoS One 11:e0154481.

Krull, A. C., J. K. Shearer, P. J. Gorden, V. L. Cooper, G. J. Phillips, and P. J. Plummer. 2014. Deep sequencing analysis reveals temporal microbiota changes associated with development of bovine digital dermatitis. Infect. Immun. 82:3359-3373.

Krull, A. C., J. K. Shearer, P. J. Gorden, H. M. Scott, and P. J. Plummer. 2016b. Digital dermatitis: Natural lesion progression and regression in Holstein dairy cattle over 3 years. J. Dairy Sci. 99:3718-3731.

Landis, J. R., and G. G. Koch. 1977. The measurement of observer agreement for categorical data. Biometrics 33:159-174.

Laven, R. A., and D. Logue. 2006. Treatment strategies for digital dermatitis for the UK. Vet. J. 171:79-88.

Manning, A. D., S. A. Mahendran, B. S. Hurst, T. L. Blackmore, and N. J. Bell. 2017. Effect of a prewash on footbath contamination: A randomised control trial. Vet. Rec. 180:121.

Manske, T., J. Hultgren, and C. Bergsten. 2002. Topical treatment of digital dermatitis associated with severe heel-horn erosion in a Swedish dairy herd. Prev. Vet. Med. 53:215-231.

Moore, D. A., S. L. Berry, M. L. Truscott, and V. Koziy. 2001. Efficacy of a nonantimicrobial cream administered topically for treatment of digital dermatitis in dairy cattle. J. Am. Vet. Med. Assoc. 219:1435-1438.

Mumba, T., D. Döpfer, C. Kruitwagen, M. Dreher, W. Gaastra, and B. A. M. d. Zeijst. 1999. Detection of spirochetes by polymerase chain reaction and its relation to the course of digital dermatitis after local antibiotic treatment in dairy cattle. Zentralbl Veterinarmed B. $46: 117-126$.

Nielsen, B. H., P. T. Thomsen, L. E. Green, and J. Kaler. 2012. A study of the dynamics of digital dermatitis in 742 lactating dairy cows. Prev. Vet. Med. 104:44-52.

Nishikawa, A., and K. Taguchi. 2008. Healing of digital dermatitis after a single treatment with topical oxytetracycline in 89 dairy cows. Vet. Rec. 163:574-576.

Nordhoff, M., A. Moter, K. Schrank, and L. H. Wieler. 2008. High prevalence of treponemes in bovine digital dermatitis-A molecular epidemiology. Vet. Microbiol. 131:293-300.
Oliveira, V. H. S., J. T. Sorensen, and P. T. Thomsen. 2017. Associations between biosecurity practices and bovine digital dermatitis in Danish dairy herds. J. Dairy Sci. 100:8398-8408.

Orsel, K., P. Plummer, J. Shearer, J. De Buck, S. D. Carter, R. Guatteo, and H. W. Barkema. 2017. Missing pieces of the puzzle to effectively control digital dermatitis. Transbound. Emerg. Dis. https://doi.org/10.1111/tbed.12729.

Potterton, S. L., N. J. Bell, H. R. Whay, E. A. Berry, O. C. D. Atkinson, R. S. Dean, D. C. J. Main, and J. N. Huxley. 2012. A descriptive review of the peer and non-peer reviewed literature on the treatment and prevention of foot lameness in cattle published between 2000 and 2011. Vet. J. 193:612-616.

Relun, A., R. Guatteo, P. Roussel, and N. Bareille. 2011. A simple method to score digital dermatitis in dairy cows in the milking parlor. J. Dairy Sci. 94:5424-5434.

Relun, A., A. Lehebel, N. Bareille, and R. Guatteo. 2012. Effectiveness of different regimens of a collective topical treatment using a solution of copper and zinc chelates in the cure of digital dermatitis in dairy farms under field conditions. J. Dairy Sci. 95:3722-3735.

Rodriguez-Lainz, A., P. Melendez-Retamal, D. W. Hird, and D. H. Read. 1998. Papillomatous digital dermatitis in Chilean dairies and evaluation of a screening method. Prev. Vet. Med. 37:197-207.

Schulz, K. F., and D. A. Grimes. 2002. Unequal group sizes in randomised trials: Guarding against guessing. Lancet 359:966-970.

Shearer, J. K., and J. Hernandez. 2000. Efficacy of two modified nonantibiotic formulations (Victory) for treatment of papillomatous digital dermatitis in dairy cows. J. Dairy Sci. 83:741-745.

Solano, L., H. W. Barkema, C. Jacobs, and K. Orsel. 2017a. Validation of the M-stage scoring system for digital dermatitis on dairy cows in the milking parlor. J. Dairy Sci. 100:1592-1603.

Solano, L., H. W. Barkema, S. Mason, E. A. Pajor, S. J. LeBlanc, and K. Orsel. 2016. Prevalence and distribution of foot lesions in dairy cattle in Alberta, Canada. J. Dairy Sci. 99:6828-6841.

Solano, L., H. W. Barkema, C. Pickel, and K. Orsel. 2017b. Effectiveness of a standardized footbath protocol for prevention of digital dermatitis. J. Dairy Sci. 100:1295-1307.

Somers, J. G. C. J., K. Frankena, E. N. Noordhuizen-Stassen, and J. H. M. Metz. 2005. Risk factors for digital dermatitis in dairy cows kept in cubicle houses in The Netherlands. Prev. Vet. Med. $71: 11-21$.

Stokes, J. E., K. A. Leach, D. C. J. Main, and H. R. Whay. 2012. The reliability of detecting digital dermatitis in the milking parlour. Vet. J. 193:679-684.

Tang, K. L., N. P. Caffrey, D. B. Nóbrega, S. C. Cork, P. E. Ronksley, H. W. Barkema, A. J. Polachek, H. Ganshorn, N. Sharma, and J. D. Kellner. 2017. Restricting the use of antibiotics in foodproducing animals and its associations with antibiotic resistance in food-producing animals and human beings: A systematic review and meta-analysis. Lancet Planetary Health 1:e316-e327.

Thomsen, P. T., A. K. Ersbøll, and J. T. Sørensen. 2012. Short communication: Automatic washing of hooves can help control digital dermatitis in dairy cows. J. Dairy Sci. 95:7195-7199.

van Amstel, S. R., S. van Vuuren, and C. L. C. Tutt. 1995. Digital dermatitis: Report of an outbreak. J. S. Afr. Vet. Assoc. 66:177-181.

van Andel, M., T. Rawdon, K. Thompson, and D. Vink. 2012. Review of recent bovine digital dermatitis-like lesions in cattle. Surveillance (Wellington) 39:9-13.

Watson, C. 2007. Lameness in Cattle. Crowood Press, Marlborough, UK

Wells, S. J., L. P. Garber, and B. A. Wagner. 1999. Papillomatous digital dermatitis and associated risk factors in US dairy herds. Prev. Vet. Med. 38:11-24.

Winders, T., M. Socha, and G. Cramer. 2015. An evaluation of the agreement between digital dermatitis scoring methods in the parlor, pen and hoof-trimming chute. Page 134 in Proc. Int Lameness in Ruminants Conf., Valdivia, Chile. 


\section{APPENDIX: PRODUCT DETAILS}

\section{HealMax}

Manufacturer: AgroChem Inc., Saratoga Springs, New York

Proprietary blend containing 1.5 pentanedial, alcohol ethoxylate, propylene glycol, xantham gum, tocopherol (vitamin E), methyl salicylate, and water

Directions for use: HealMax spray is applied directly to hoof area. Apply 3 to $5 \mathrm{~mL}$ once or twice on either the same or consecutive days. For best results, remove excess mud and manure before application. Do not wrap hoof. Patent pending (no. 61/200,367).

\section{Intra HoofSol Liquid}

Manufacturer: Diamond Hoof Care Ltd., Intracare BV, Veghel, the Netherlands

Active ingredients: copper sulfate pentahydrate $15.2 \%$, zinc sulfate monohydrate $11.4 \%$, aloe vera $6.4 \%$. Nonactive ingredients: distilled water, citric acid monohydrate, xanthan gum, lutein as coloring.
Directions for use: Use the head lockers in the feeding alley or the milking parlor to securely hold the animals. Works great in stanchion barns as well. If necessary, rinse the hooves and interdigital spaces with water. Premium coverage and results: apply weekly at $50 \%$ solution. Optional alternative: use a reduced dilution (20-40\% Hoof-Sol Liquid) for weekly follow-up sessions to maintain hoof health. Spray the Hoof-Sol Liquid on the back and in the interdigital spaces of the hind legs.

\section{Tetracycline $250(250 \mathrm{mg} / \mathrm{g})$}

Manufacturer: Vetoquinol Inc., Lavaltrie, Quebec, Canada

Description: Tetracycline HCI is an antibiotic similar to oxytetracycline and chlortetracycline in bacterial spectrum. Tetracycline is rapidly absorbed and effective in the treatment of many diseases resulting from gram-positive and gram-negative bacteria, spirochetes, rickettsia, and some chlamydia. Drug Identification Number 00527777. 